\title{
OptoSci educator kits: an immediate solution to photonics teaching laboratories
}

Douglas Walsh, David Moodie, lain Mauchline, Walter Johnstone, Brian Culshaw

Douglas Walsh, David Moodie, lain Mauchline, Walter Johnstone, Brian Culshaw, "OptoSci educator kits: an immediate solution to photonics teaching laboratories," Proc. SPIE 9663, Eighth International Topical Meeting on Education and Training in Optics and Photonics, 96632C (6 October 2003); doi: $10.1117 / 12.2207510$

Event: Eighth International Topical Meeting on Education and Training in Optics and Photonics, 2003, Tucson, Arizona, United States 


\title{
OptoSci Educator Kits - an Immediate Solution to Photonics Teaching Laboratories
}

\author{
Douglas Walsh, David Moodie and Iain Mauchline \\ OptoSci Ltd, 141 St. James Rd., Glasgow, G4 OLT, Scotland \\ www.optosci.com T: +44 141552 7020,F: +44141552 3886, E: info@optosci.com \\ Walter Johnstone and Brian Culshaw \\ EEE Dept., University of Strathclyde, 204 George St., Glasgow, G1 1XW, Scotland
}

\begin{abstract}
The burgeoning growth of the worldwide photonics and optical communications industry has imposed ever increasing demands on the supply of suitably skilled engineers and scientists who can design, install and operate modern photonics systems. In recognition of this need OptoSci, in collaboration with university academics, has commercially developed a series of hardware based teaching packages in optics, optoelectronics and optical communications. Each educator kit is fully self-contained, including all of the optoelectronic hardware and comprehensive literature support. This saves the academic tutor considerable development time and enables the kits to be immediately installed in the photonics teaching laboratory to support accompanying lecture courses. A fundamental design objective of our educator kits is to provide students with hands-on practical experience of photonics components, instruments and systems and allow them to investigate essential physical principles and key technical issues relevant to their lecture courses. This paper will outline the design philosophy behind the products to meet the desired educational aims, and then examine the specific educational objectives and topics investigated in each educator kit.

(C) 2003 Optical Society of America

OCIS codes: (000.2060) Education: (060.2330) Fiber optics communications; (060.4510) Optical communications; (230.7390)

Waveguides, planar; (260.0260) Physical optics; (140.3510) Lasers, fiber; (060.2320) Fiber optics amplifiers and oscillators; (060.2410) Fibers, erbium
\end{abstract}

\section{Introduction}

Photonics technology currently drives major technical advancement in a wide diversity of technologies such as telecommunications, measurement science, industrial and environmental sensing, medical diagnostics and biosciences. In particular, the world's main trunk telecommunications systems, the global internet and mobile phone communications systems are all founded on Photonics networks. Companies operating in these fields have an ever increasing demand for highly skilled scientists and engineers who can design, build, analyse, install and operate photonics systems. There is no doubt that the learning experience of these professional technologists is greatly enhanced during their graduate or undergraduate studies by exposure to hands on, practical experience of photonics components and systems. OptoSci Ltd, in collaboration with academics at Strathclyde and Heriot-Watt Universities, has developed a suite of Photonics Educator Kits which enable students to experimentally investigate all of the major technical features, principles and design issues of optical waveguides, optical communications systems, optical networks and OTDR, erbium doped fibre amplifiers and lasers. These application oriented kits are also supported by a range of experiments examining the fundamentals of physical optics, covering reflection, refraction, polarisation, diffraction, coherence and interference. In the development of all of these systems we adhere to a strict design philosophy and procedure, which ensures that all of the important educational objectives are met.

\section{Design Philosophy}

The overall educational aims of the experimental exercises are to enable students to consolidate their understanding and knowledge of photonics as presented in an accompanying lecture course and to acquire practical experience of the design, analysis and characteristics of photonics components and systems. To achieve these aims it is essential to take a fully integrated approach to the design of laboratory based photonics teaching packages including the design of dedicated hardware, experimental procedures, exercises and manuals. To ensure that all desirable educational objectives are met and that all of the most important scientific and technical principles, issues and 
phenomena are addressed, we have developed our suite of fully integrated laboratory based teaching packages in accordance with the following design rules:

- Define the educational objectives in terms of the physical principles, important technical features, design issues and performance characteristics which must be addressed, with particular attention to facilitating student understanding and ability to implement concepts.

- Define the experiments to meet these performance objectives.

- Design the dedicated (custom) hardware to enable the proposed experimental investigation whilst keeping costs within realistic academic teaching budgets.

- Formulate the experimental procedure and manuals to guide the students through the investigation and results analysis (in some cases more open ended investigations may be formulated with minimal guidance to the students).

- Formulate tutorial exercises and case studies to relate the results to real world devices and systems.

The primary constraint is cost and the final packages must be affordable within higher education budgets. In general, the packages have been designed as far as possible to be self-contained so that as little ancillary equipment as possible is required. However, where it is advantageous and cost effective to use equipment normally available in student laboratories, the packages have been designed to be compatible with the capabilities of such equipment e.g. a $20 \mathrm{MHz}$ or $50 \mathrm{MHz}$ oscilloscopes.

\section{Photonics Educator Kits}

Using the design principles referred to above, OptoSci has commercially developed a unique range of fully selfcontained laboratory based teaching packages for use in universities, colleges, and industrial training centres. The current series of educator kits allow students to perform detailed experimental investigations of the following topics:

$\begin{array}{ll}\text { Optical Waveguiding } & \text { Physical Optics } \\ \text { Fibre Optic Communications } & \text { Optical Network Analysis - OTDR } \\ \text { Erbium Doped Fibre Amplifiers } & \text { Lasers }\end{array}$

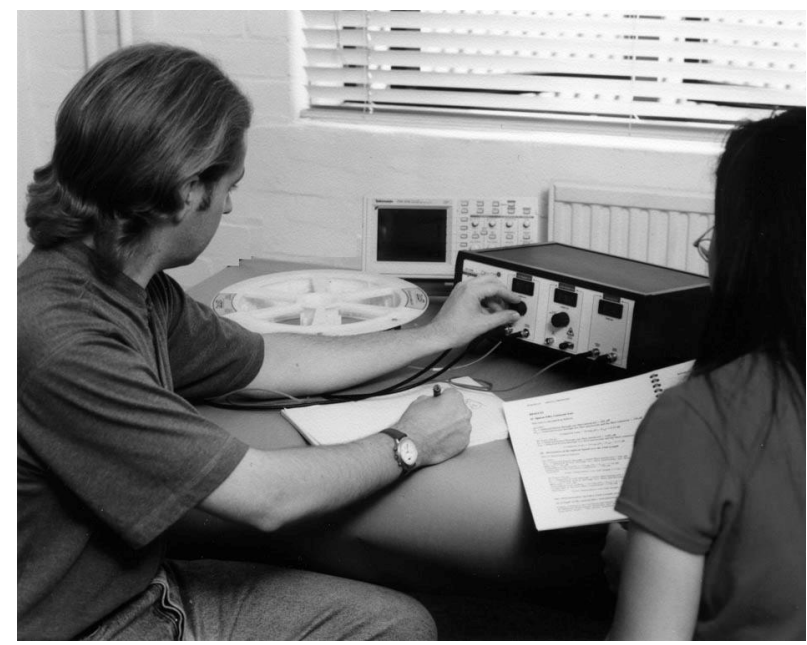

Figure 1: An educator kit at work!

The kits are designed in conjunction with senior academics from the internationally renowned optoelectronics teaching and research groups at Strathclyde and Heriot-Watt Universities to ensure high quality products that are directly relevant to teaching or training courses in this technological field. Furthermore, each package is a fully selfcontained unit, incorporating all of the components and optoelectronic instruments required to perform the experiments thus allowing each kit to be immediately installed in the teaching laboratory. In addition, each educator kit is supplied with extensive literature support for the tutor and student. This includes, student manuals which describe the relevant background theory and experimental procedures; instructor's manual with sample results for all 
experiments and exercises; detailed lecture notes with case studies and design exercises, and a series of tutorial questions with solutions.

The table below summarises some of the features and benefits of this range of teaching packages

\begin{tabular}{|c|c|c|}
\hline FEATURES & BE & NEFITS \\
\hline Fully self contained package & $\Rightarrow$ & $\begin{array}{l}\text { Saves } 2 \text { to } 3 \text { years of course, literature and } \\
\text { hardware development }\end{array}$ \\
\hline Competitively priced & $\Rightarrow$ & $\begin{array}{l}\text { Available for a price which is realistic within } \\
\text { academic budgets }\end{array}$ \\
\hline All specialised experimental hardware is supplied & $\Rightarrow$ & $\begin{array}{l}\text { Allows immediate installation in the } \\
\text { laboratory }\end{array}$ \\
\hline Comprehensive laboratory literature support & $\Rightarrow$ & $\begin{array}{l}\text { Full background and experimental support } \\
\text { for tutor and student }\end{array}$ \\
\hline Full lecture notes and tutorials provided & $\Rightarrow$ & $\begin{array}{l}\text { Provides extensive background material for } \\
\text { lecture course }\end{array}$ \\
\hline Designed in conjunction with leading academics & $\Rightarrow$ & $\begin{array}{l}\text { Totally relevant to photonics courses in } \\
\text { academia }\end{array}$ \\
\hline $\begin{array}{l}\text { Easily tailor experimental programme for different } \\
\text { student levels }\end{array}$ & $\Rightarrow$ & $\begin{array}{l}\text { Suitable for all undergraduate and masters } \\
\text { level photonics courses in Physics and } \\
\text { Electronic Engineering }\end{array}$ \\
\hline Straightforward to reconfigure for open ended projects & $\Rightarrow$ & $\begin{array}{l}\text { Can also be used for project based } \\
\text { experiments }\end{array}$ \\
\hline Innovative design philosophy & $\Rightarrow$ & $\begin{array}{l}\text { Ensures that all desired educational } \\
\text { objectives are realised and that students } \\
\text { investigate all major technical issues }\end{array}$ \\
\hline $\begin{array}{l}\text { Hundreds of kits are currently used in leading academic } \\
\text { institutions world-wide and we experience continued } \\
\text { repeat business }\end{array}$ & $\Rightarrow$ & $\begin{array}{l}\text { Positive endorsement of the educational } \\
\text { value of the products by both tutors and } \\
\text { students }\end{array}$ \\
\hline Full product support is available & $\Rightarrow$ & Just contact us by e-mail, phone, or fax \\
\hline
\end{tabular}

Table 1: Features \& Benefits of OptoSci's Photonics Educator Kits

Based on the many benefits that our range of photonics educator kits offer and the very positive feedback we have obtained from students and lecturers alike, we believe that these innovative products offer the tutor an immediate and cost effective solution to the provision of comprehensive and stimulating experimental courses in key areas of optics, optoelectronics, and optical communications.

The following sections provide a summary of the key educational objectives for each of the teaching packages and then briefly describe the associated experimental programme used to achieve these aims. A more detailed description of some of the background theory, experimental hardware, results and analysis is provided in previous publications [1-3] and on the OptoSci website [4].

\section{Optical Waveguiding}

Information transmission along an optical fibre is governed by the principles and characteristics of optical waveguiding. The simplest approach to introducing students to the concepts and properties of optical waveguides is to begin with the principles of total internal reflection and then address the ray model of firstly step index, and then graded index planar waveguides. To support lecture courses on these topics, the overall objectives of the Optical Waveguiding package are to enable students to experimentally investigate and consolidate their understanding and knowledge of: 
- the principles of refraction, reflection and total internal reflection.

- the principles of optical waveguiding using the ray model and the concept of guided modes.

- the principles and practice of the prism coupling technique for the measurement of modal parameters and the investigation of mode spectra, and as an illustration of phase matching.

- elementary waveguide analytical techniques.

- basic waveguide design processes including concepts of mode cut-off and the design of single mode waveguides.

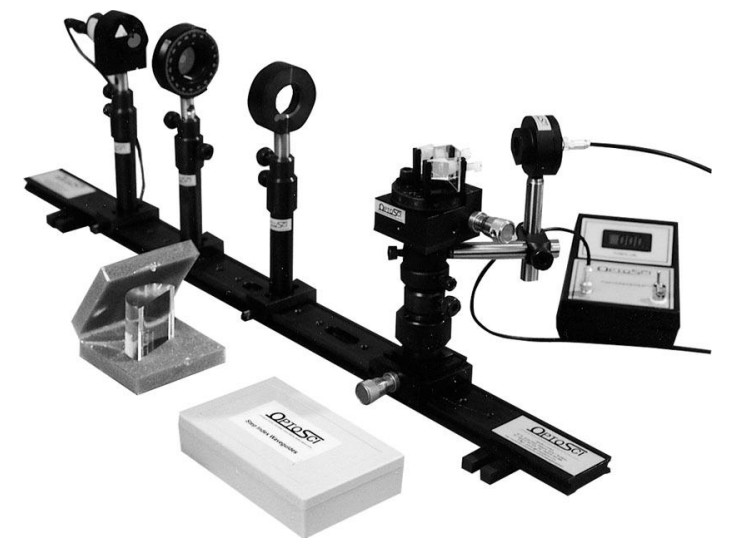

Figure 2: Optical waveguiding educator kit

To meet the educational objectives stated above the students carry out the following investigation:

- Measurement of Snell's law.

- Measurement of the Fresnel relationships for both polarisation states with observations of Brewster's angle, the critical angle and total internal reflection (comparisons are carried out with theory).

- Establishment of prism coupling to selective waveguide modes and observation of output coupled mode lines ( $\mathrm{m}$ line spectra).

- Measurement of mode coupling angles and mode effective indices / propagation constants for

- $\quad$ Step and graded index planar waveguides.

- Both polarisation states.

- Determination of the waveguide parameters (index profiles and thickness) from the mode effective indices.

- Calculation of mode cut-off conditions using the waveguide parameters.

- Design and test of single mode waveguides.

\section{Fibre Optic Communications}

Optical fibre information transmission links enable more information to be transmitted over greater distance than any other communications technology. Hence, they have all but completely replaced copper based systems as the primary choice for global and local telecommunications systems. The objectives of the Optical Communications experiments are to enable students to experimentally investigate and build upon their knowledge and conceptual understanding of, and their ability to interpret:

- The main characteristics of the major components of a fibre optic communications system i.e. the source / transmitter, the fibre channel (attenuation, dispersion, pulse spreading etc.) and the receiver

- The overall system performance limitations imposed by the component characteristics

- the maximum possible link length limited by attenuation

- the bit rate (and bandwidth) / length products determined by fibre dispersion

- System design and performance analysis. 


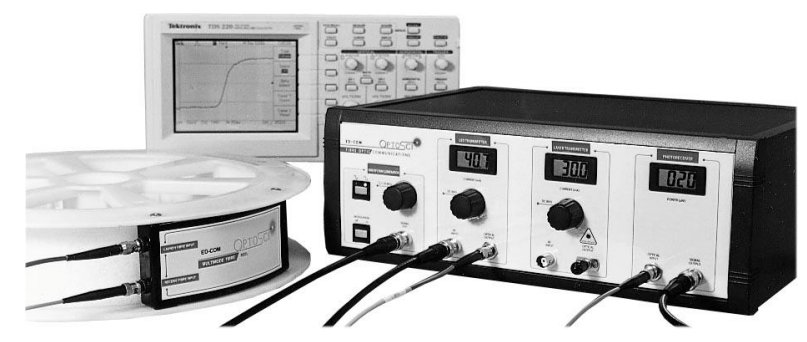

Figure 3: Fibre optic communications educator kit

To achieve these objectives the students carry out the following investigations:

Stage 1. Power Budgets

- Measurement of the power / current characteristics, bias points and launched powers of the laser and LED transmitters.

- Measurement of connector losses.

- Measurement of the fibre attenuation coefficient.

- Measurement of the receiver noise and sensitivity.

- Calculation and comparison of the attenuation limited link lengths for the laser and LED transmitters.

Stage 2. Temporal Characteristics

- Measurement of the step function response of the transmitter / receiver, the system and the fibre using both the laser and the LED. This enables the determination of

- the fibre impulse response for both the laser and the LED

- the modal and material dispersion coefficients

- the bit rate distance products for both the laser and LED transmitters.

- Measurement of the analogue signal frequency response of the transmitter / receiver, the system and the fibre, leading to determination of

- the analogue bandwidth and bandwidth . distance products of the fibre for both the LED and laser sources. It is interesting to compare the directly measured bandwidth with that obtained from the step response

- Measurement of the impulse response with direct determination of the fibre dispersion coefficients.

Stage 3. System Performance and Analysis

- The design of systems to meet a given specification using the measured data.

- Analysis of the performance of systems to determine if they will meet a required specification.

- Design and performance analysis for state of the art systems at $1.3 \& 1.55 \mu \mathrm{m}$ to compare with the results for the system investigated.

\section{Erbium Doped Fibre Amplifiers And Lasers}

Direct optical amplification using erbium doped fibre amplifiers (EDFAs) is now preferred over optoelectronic repeaters as the primary means of restoring the signal power in long distance fibre optic links and branched networks. In addition, lasers (essentially optical oscillators) are simply optical amplifiers with positive feedback, again highlighting the importance of optical amplifiers in modern photonics systems. The objectives of the EDF optical amplifier and laser experiments are to enable students to investigate and become practically familiar with the principles and characteristics of optical amplifiers and lasers in general, and erbium doped fibre amplifiers and lasers in particular. To achieve these objectives the EDF amplifier and laser experiments enable:

- Measurement and analysis of small and large signal gain as a function of pump power

- Measurement of gain as a function of signal power and pump power 
- Investigation of gain saturation and determination of point of transparency, gain gradient and gain efficiency

- Determination of saturated output power as a function of pump power

- Investigation of amplified spontaneous emission (ASE) and ASE-ASE and Signal-ASE beat noise. This includes a study of their dependencies on pump and signal power

- Determination of output signal to noise ratio and Noise Figure for the EDFA

- Construction of an EDF laser

- Examination of single pass amplifier gain characteristics

- Investigation of the laser output power characteristic (threshold and slope efficiency) as a function of the output coupling ratio and the intra-cavity loss.

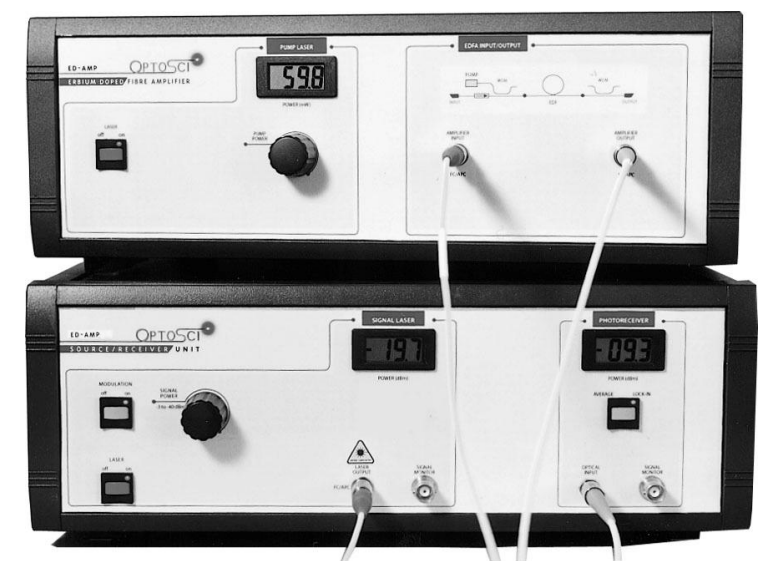

Figure 4: Erbium doped fibre amplifier educator kit

\section{Optical Network Analysis - OTDR}

In optical fibre networks, signal losses occur in the fibre itself, at splices and connectors and in the excess loss mechanisms within components like couplers and wavelength division multiplexers. With the passage of time, faults, such as fibre breaks, may occur, and splices, connectors and components degrade, resulting in increasing transmission losses which jeopardise the system performance. Optical time domain reflectometry (OTDR) is the industry standard technique employed for measuring the loss characteristics of a fibre link or network, monitoring the network status and locating faults and degrading components. Hence the main objectives of the network analysis laboratory exercises are the investigation and practical familiarisation with:

- Network configurations

- $\quad$ point to point, branched and WDM networks

- The principles and characteristics of network components

- connectors, splices, couplers and WDMs

- The use of an optical time domain reflectometer (OTDR) including trace acquisition and manipulation

- OTDR trace analysis, feature identification and component / fibre loss assessment

- Fault identification and location. 


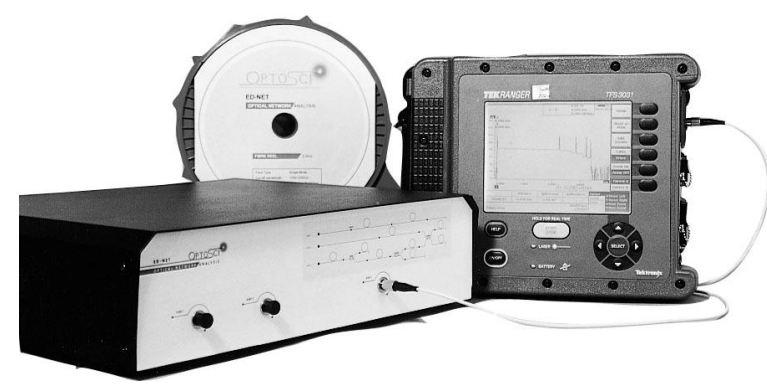

Figure 5: Optical network analysis - OTDR educator kit

The students carry out the following investigation in four stages to build up skills and knowledge towards the analysis of complex networks:

Stage 1. OTDR trace acquisition and analysis for point to point links at both $1.3 \mu \mathrm{m}$ and $1.55 \mu \mathrm{m}$

- OTDR operation and functions

- trace acquisition, cursor controls and zoom functions

- Identification of trace features and loss events: dead zones, Fresnel reflections, loss events and ghost reflections

- Measurement of distances and losses at events (splices, connectors, faults etc.)

- Measurement of the fibre attenuation coefficient and its wavelength sensitivity $(1.3 \mu \mathrm{m} \& 1.55 \mu \mathrm{m})$.

- Measurement of bend losses and their wavelength sensitivity $(1.3 \mu \mathrm{m} \& 1.55 \mu \mathrm{m})$.

Stage 2. Branched networks with fibre couplers - Coupler loss analysis

- Measurement and interpretation of losses across a fused fibre coupler event.

- Estimation of the coupler insertion and excess losses from the OTDR trace loss measurements (given knowledge of the coupling ratio, $\mathrm{K}$ ).

- Estimation of the coupling ratio, $\mathrm{K}$, from the loss measurements.

- Determination of the wavelength sensitivity of K $(1.3 \mu \mathrm{m} \& 1.55 \mu \mathrm{m})$.

Stage 3. WDM networks with multiple fibre coupler branches

- Trace acquisition and investigation of the $1.3 \mu \mathrm{m} \& 1.55 \mu \mathrm{m}$ branches of a WDM network.

- Measurement of WDM insertion loss and isolation.

- Detailed investigation of the $1.55 \mu \mathrm{m}$ branch beyond the WDM with analysis of coupler losses.

Stage 4. Fault location and identification on networks with deliberately introduced faults

- Identification of line faults and determination of their losses.

- Identification and loss analysis of faults at couplers and WDMs - coupler degradation or splice degradation and its location.

- Identification, location (distance and which branch?) and loss analysis of line faults in particular branches of a multi branch network.

\section{Physical Optics}

The Principles of Physical Optics educator kit addresses the fundamental properties of light and the principles of physical optics. It consists of four individual modules covering detailed experiments in polarisation, reflection and refraction, diffraction, interference and coherence. The objective of this suite of modules is to provide the grounding in some of the basic properties of light, which are then applied in some of the kits described previously.

The students carry out the following experiments

\subsection{Polarisation}

- Confirmation of Malus's law. 
- Investigation of the properties of half and quarter wave plates (alignment, axes identification, polarisation characteristics).

- Measurement of the state of polarisation of a light wave.

- Investigation of quarter and arbitrary waveplates (Stokes parameters, the polarisation ellipse and the Poincaré sphere).

- Examination of strain birefringence and its application to strain sensing.

\subsection{Reflection And Refraction}

- Reflection and refraction characteristics at an internal and external optical interface for both $s$ - and $p$ polarisation states.

- Confirmation of Snell's Law at low to high index and high to low index optical interfaces.

- Confirmation of the Fresnel Equations

- Identification of features such as Brewster's Angle and the critical angle for total internal reflection.

- Determination of the refractive index of an optical element.

\subsection{Diffraction}

- Investigation of near and far field diffraction patterns for apertures and slits of various dimensions (Fraunhofer and Fresnel diffraction).

- Confirmation of the width of various known slits and apertures and determination of the width of unknown slits and apertures.

- Experimental investigation of diffraction at a reflective grating, including the basic grating equation (confirmation of grating line density).

- Multiple order diffraction, the Littrow configuration, grating resolution and resolving power as a function of incidence angle and diffraction order using two wavelengths.

- Determination of the wavelength of a second laser.

- Diffraction through a transmission grating and measurement of line spacing.

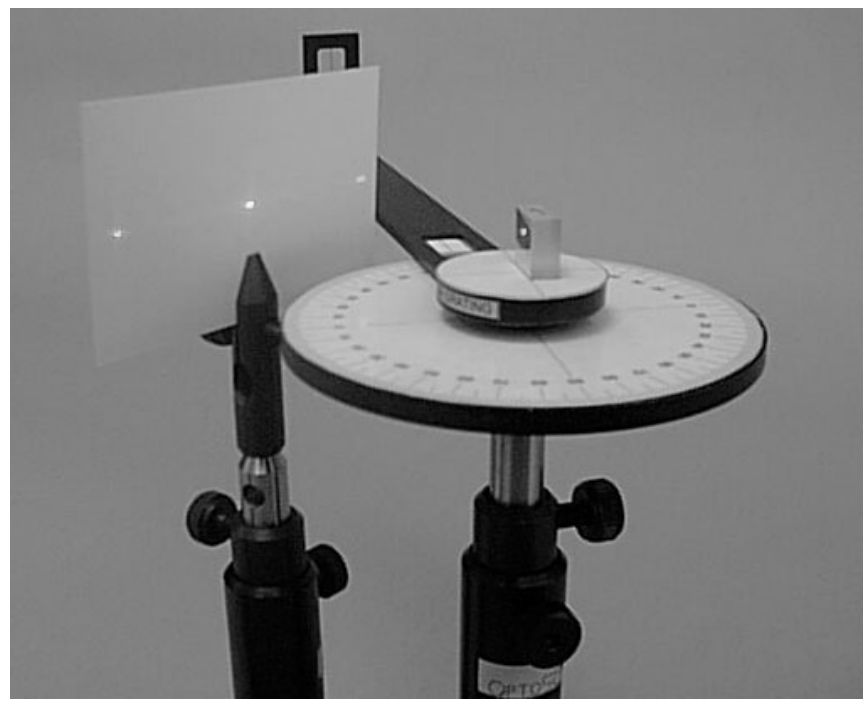

Figure 6: Diffraction module

\subsection{Interference And Coherence}

- Construction of a Michelson interferometer and investigation of its multiple and single fringe alignment configurations.

- Assessment of the surface quality of three different optical elements inserted into one arm of the interferometer.

- Calculation of the wedge angle for one of the elements.

- Investigation of the coherence function of a Fabry-Perot cavity laser.

- Examination of the coherence length of the Fabry-Perot cavity laser as the laser's drive current is varied.

- Determination of the coherence length of the laser and measurement of its cavity length. 


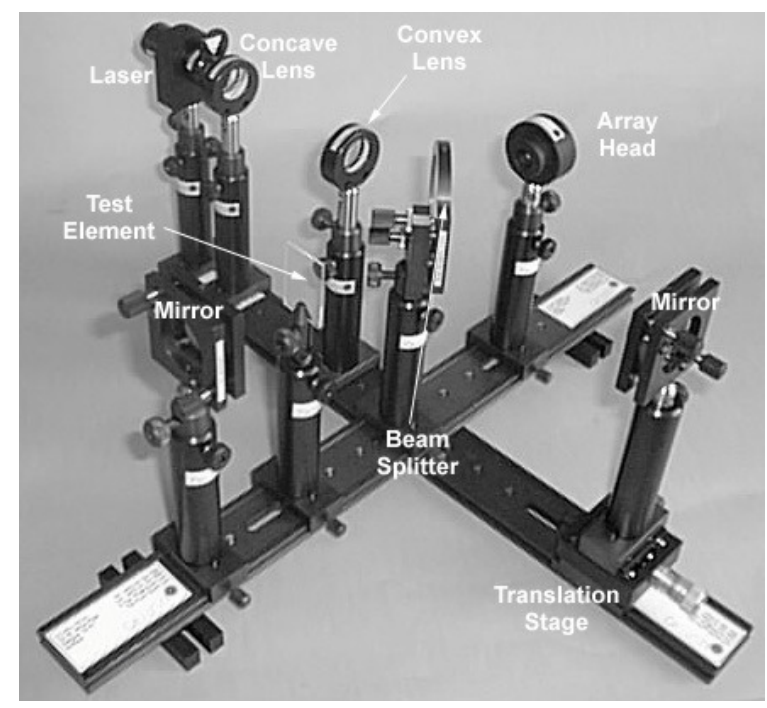

Figure 7: Interference \& coherence module

\section{Conclusions}

A suite of laboratory based experimental teaching packages has been developed for modern optics, photonics and optical communications courses. OptoSci's innovative design philosophy ensures: that all of the desired educational objectives are realised; that all major technical issues are addressed; and that each complete package can be offered for a price which is realistic within academic budgets. The kits are suitable for both physics and engineering based courses since they address fundamental physical principles, key technical issues, component and system performance characteristics and design processes (many of which, such as dispersion in optical fibres, were hitherto precluded by cost from the teaching environment). Furthermore, since each educator kit is fully self-contained the tutor is provided with all of the experimental equipment and literature support to immediately establish a teaching laboratory in key areas of photonics technology, in addition to providing extensive teaching material for the associated lecture course. Ultimately then, the key benefit for the tutor is that each of these unique and comprehensive teaching packages saves two to three years of course, literature and hardware development effort.

\section{References}

1. W. Johnstone, B. Culshaw, D. Walsh, D. Moodie and I. Mauchline, "Photonics laboratory experiments for modern technology based courses", IEEE Proceedings: Special issue on Electrical and Computer Engineering Education, pp41-54, 1999.

2. W. Johnstone, B. Culshaw, D. Walsh, D. Moodie and I. Mauchline, "Student laboratory experiments on erbium doped fibre amplifiers and lasers", 6th international conference on Education and Training in Optics and Photonics (ETOP) Cancun 1999, and SPIE Proceedings 3831-47, 2000.

3. W. Johnstone, B. Culshaw, D. Moodie, I. Mauchline and D. Walsh, "Photonics laboratory teaching experiments for scientists and engineers" 7th international conference on Education and Training in Optics and Photonics (ETOP), Singapore 2001, and SPIE Proceedings 4588-51, 2002.

4. Extensive additional information on OptoSci's range of photonics educator kits (i.e. full data sheets, a sample student manual, detailed educator kit specifications, and journal publications on the products) is available in the "Product Support" section of our website www.optosci.com. 